\title{
Reducing the quadratic divergence in the Higgs boson mass squared without top partners
}

\author{
Sonia El Hedri, ${ }^{1}$ Ann E. Nelson, ${ }^{2}$ and Devin G. E. Walker ${ }^{3}$ \\ ${ }^{1}$ NIKHEF, Theory Group, Science Park 105, 1098 XG, Amsterdam, Netherlands \\ ${ }^{2}$ Department of Physics, Box 1560, University of Washington, Seattle, Washington 98195-1560, USA \\ ${ }^{3}$ Department of Physics and Astronomy, Dartmouth College, Hanover, New Hampshire 03755, USA
}

(Received 24 May 2018; published 20 August 2018)

\begin{abstract}
We examine a model with multiple scalar fields to see whether it is possible to reduce the fine-tuning of the Standard Model Higgs mass without introducing low scale top partners. Our approach may be regarded as a generalization of the condition proposed by Veltman, who attempted to predict the Higgs mass using the criterion that the various low energy contributions to the quadratic divergence of the Higgs mass cancel. Although the Veltman condition predicts the wrong Higgs mass in the Standard Model, it can still be adapted to extended Higgs sectors. Furthermore, theories with additional Higgs bosons can lead to suppressed Yukawa couplings of the top quark to the $125 \mathrm{GeV}$ Higgs, making the associated one-loop divergence smaller. Here, we review possible extensions of the Standard Model where the Veltman condition could be realized, and study in detail one minimal model with two extra scalar fields. For this model and for a cutoff of $5 \mathrm{TeV}$, we show that the overall fine-tuning can be considerably lowered without introducing low-scale Landau poles, albeit the Higgs sector will contain fairly large couplings at the cutoff. Models where the top Yukawa coupling is reduced, in particular, will be within the reach of the upcoming LHC searches.
\end{abstract}

DOI: $10.1103 /$ PhysRevD.98.035029

\section{INTRODUCTION}

The discovery of the Higgs boson at the Large Hadron Collider (LHC) [1,2] is a triumph for particle physics, as it was one of the last missing pieces needed to understand the origin of the masses of the Standard Model (SM) particles. Although the SM is now consistent up to high scales, some aspects of the theory still appear contrived. In particular, the mass of the Higgs boson, $125 \mathrm{GeV}$, is sensitive to physics at much higher scales. At one-loop, the SM Higgs mass squared receives sizable corrections that depend quadratically on the cutoff energy scale, $\Lambda$, as follows:

$$
m_{h}^{2}=m_{0}^{2}+\frac{1}{16 \pi^{2}}\left(\frac{3}{4} g_{1}^{2}+\frac{9}{4} g_{2}^{2}+3 \lambda_{h}-12 \lambda_{t}^{2}\right) \Lambda^{2},
$$

where $\lambda_{h}, \lambda_{t}, \mathrm{v}, g_{2}$, and $g_{1}$ are the Higgs quartic, top Yukawa, electroweak vacuum expectation value (VEV), $S U(2)_{L}$, and hypercharge gauge couplings, respectively. $m_{0}$ is the bare Higgs mass parameter that appears in the Lagrangian prior to renormalization, and $m_{h}$ is the

Published by the American Physical Society under the terms of the Creative Commons Attribution 4.0 International license. Further distribution of this work must maintain attribution to the author(s) and the published article's title, journal citation, and DOI. Funded by SCOAP ${ }^{3}$. renormalized quadratic term which determines the value of the physical Higgs mass. $\Lambda$ is a momentum cutoff, presumably the next fundamental scale of new physics. In the minimal Standard Model, with a cutoff of $\Lambda \sim 5 \mathrm{TeV}$, the value of $m_{0}$ must be fine-tuned at the level of one part in 100. This hefty dependence of the physical Higgs mass on the cutoff requires a cancellation between physics at the cutoff scale against physics below the cutoff scale which is at odds with the expectation that physics at low energies should not be highly sensitive to the short distance theory $[3,4]$. Before the discovery of the Higgs boson, accompanied by the lack of discovery of supersymmetry or any other mechanism for canceling quadratic divergences, it had been expected that the scale of new physics beyond the $\mathrm{SM}$ would be around or lower than a $\mathrm{TeV}$ in order to avoid fine-tuning the SM parameters. The LHC, however, can directly probe much higher energy scales, as high as several $\mathrm{TeV}$, and has discovered no new physics beyond the SM. Moreover precision electroweak measurements, which favored a light SM Higgs boson, imply that $\Lambda$ is likely large $(\Lambda \gtrsim 5 \mathrm{TeV})[5,6]$ if the new physics particles have electroweak quantum numbers and couple to the SM Higgs.

This gap between the scale of new physics implied by avoidance of fine-tuning and the scale of new physics forecasted by precision electroweak measurements is known as the little hierarchy problem (LHP). The traditional way to address the LHP has been to add new particles 
and symmetries to the SM to ensure the one-loop quadratic divergence in Eq. (1) is canceled [7] without large precision electroweak corrections. Notably, since the largest divergence is generated by top quarks, extending the SM with fermionic or bosonic top partners is sufficient to quantitatively solve the LHP $[8,9]$. Introducing such top partners has thus played a large role in theoretical particle physics for the past 35 years. Prominent examples include extradimensions [10-13], intermediate Higgs (rebranded in the literature as natural composite Higgs) [8], little Higgs [14], twin Higgs [15], and supersymmetry [9,16]. In order for the cancellations to be effective in these models, though, the top partners must have a mass not too far above the top quark mass. However, the LHC has placed such strong lower bounds on the masses of colored top partners [17] that many of the scenarios listed here are now fine-tuned in most of their parameter space.

While the colored top partners are currently under siege, an extended Higgs sector is still relatively unconstrained by the LHC. Although the Higgs couplings to gauge bosons have been measured with high precision, the uncertainties on its couplings to fermions, notably to top quarks, are relatively large [18-20]. In the light of these results, it is particularly tempting to look for a solution to the finetuning problem in either or both of these blind spots. In what follows, we study how to exploit these uncertainties to cancel or at least alleviate the fine-tuning of the Higgs boson mass.

\section{A. Parametric naturalness}

Instead of the divergence structure shown in Eq. (1), we consider a scenario where the largest one-loop corrections to the Higgs mass has the following form [21]:

$m_{h}^{2}=m_{0}^{2}+\frac{1}{16 \pi^{2}}\left(\frac{3}{4} g_{1}^{2}+\frac{9}{4} g_{2}^{2}+3 \lambda_{h}-12 \lambda_{t}^{2}+\sum_{i} c_{i} \lambda_{i}\right) \Lambda^{2}$.

Here for simplicity we assume a common cutoff scale, $\Lambda$. The $\lambda_{i}$ typically correspond to new quartic couplings, present in extended Higgs sectors. Given the cutoff $\Lambda>m_{h}$, there are several strategies to make $m_{h}$ naturally small:

(1) The $\lambda_{i}$ summation can be chosen so that the parameters in the last term of Eq. (2) sum to a small number. The physical mass, $m_{h}$, is mostly set by the bare mass, $m_{0}$.

This requirement is known as Veltman's condition [22]. Veltman, inspired by Wilsonian effective field theories [3,4], insisted the dimensionless parameters in Eq. (1) sum to zero. This requirement predicted a $316 \mathrm{GeV}$ SM Higgs mass for the minimal SM, which is not realized in nature. In order to balance the top Yukawa coupling contribution in Eq. (2), in a renormalizable theory at least some of the new particles running in the loop must be bosonic so that the coefficients $c_{i}$ are positive. New gauge bosons with electroweak quantum numbers are generically constrained by the LHC to be at least multi-TeV in mass [23-25]. The LHC constraints on new scalars, although weaker, can still push their masses up to almost a TeV. When the scalar masses are set by their quartic couplings and VEVs alone, this result in turn leads to Landau poles well before the LHP can be solved $[26,27]$. In order to ensure a sizable separation between the new and SM Higgs masses, we therefore consider models involving dimensionful trilinear couplings between the different scalars. Because of gauge invariance, such models will necessarily involve not only doublets but also at least one scalar singlet or triplet that gets a VEV.

Using extended Higgs sectors to address Veltman's condition has been done in the literature in various ways. For example, Refs. [28-31] feature an extended Higgs sector with masses that generate additional quadratic divergences and hierarchy problems. For many extended Higgs sectors in the literature, it is thus impossible to eliminate all of the quadratic divergences [28-50].

We introduce two new variations of the Veltman condition, always assuming a single cutoff scale for all loops with a simple momentum cutoff:

(2) The bare mass, $m_{0}(\Lambda)$, is evaluated at the cutoff and is zero. Then the renormalized Higgs mass is set by the radiative correction and is proportional to the new cutoff scale, $\Lambda^{\prime}$.

In this scenario, it is necessary for the coefficient of $\Lambda^{2}$ in Eq. (2) to be small but not zero. The $16 \pi^{2}$ suppression helps tremendously; however, near but not complete cancellations between fermion and bosonic Higgs couplings are still required. This requirement is a mixture between Veltman's condition and an implied aim of Coleman and Weinberg to obtain electroweak symmetry breaking entirely from radiative corrections [51]. We assume that at the cutoff, the UV physics gives no contribution to $m_{0}$, and then that the $\lambda_{i}$ are such that the quantum corrections yield the observed mass of the SM Higgs at the weak scale.

The last and least restrictive variation of the Veltman condition is simply motivated by avoiding fine-tuned cancellations between physics at different scales.

(3) Both $m_{0}$ and the radiative correction are nonzero. This might seem like no constraint at all, but we will search for regions in parameter space where the cancellations between $m_{0}$ and the one-loop corrections to the Higgs mass are not finely tuned, or at least less fine-tuned than in the SM.

\section{A MINIMAL MODEL}

We focus on building a theory that addresses the LHP without adding new symmetries and partners to cancel the one-loop correction shown in Eq. (1). In part we are exploiting the fact that the top Yukawa coupling to the $125 \mathrm{GeV}$ Higgs is still not measured precisely at the LHC. The most precise direct measurement allows this coupling 
to be reduced by as much as $26 \%$ at the $95 \%$ C.L. [18-20]. This can allow for a reduction of the overall fine-tuning. It is possible that the top quark receives part of its mass from the VEV of a heavier Higgs. We thus consider a two Higgs doublet model. We also add a neutral, real scalar field, $\Phi$, in order to allow for soft trilinear scalar couplings. We consider the standard type-II two Higgs doublet model (2HDM) [52], although, since the main fermion coupling we are interested in is the top, we expect similar results in other 2HDM variants. We could also consider allowing both doublets to couple to the top quark, which could introduce flavor changing neutral scalar coupling into the up-quark sector. Since these couplings to light quarks are very small, this type of flavor changing neutral currents (FCNC) is typically compatible with the current experimental constraints.

\section{A. Requirements}

A first step when constructing a mechanism to alleviate the fine-tuning problem is to realize that, in the SM, the corrections to the Higgs mass shown in Eq. (1) are largely dominated by the top quark contributions. The size of these contributions, however, strongly depends on the size of the top Yukawa coupling to the Higgs, $\lambda_{t}$. Although this coupling is about one in the SM, it has not been precisely measured in the LHC yet. Lower Yukawa couplings would allow one to significantly reduce the amount of fine-tuning currently associated with many theories of new physics, whose energy scales have been pushed to a few $\mathrm{TeV}$ by the LHC. In what follows, we therefore investigate how simple extensions of the SM could lead to reduced top quark couplings to the Higgs and to what extent these different models would reduce the fine-tuning of the Higgs mass.

The large value of the top Yukawa coupling in the Standard Model is necessary to explain the observed top quark mass of $174 \mathrm{GeV}$. In order to reduce the large contribution to the Higgs mass radiative correction associated with this coupling, it is therefore necessary to introduce either new vectorlike fermions that mix with the top quark or new Higgs bosons that will provide additional contributions to the top mass. The first approach has already been thoroughly explored by [8]. Here, we focus on the second scenario and investigate models involving two Higgs doublets $H_{1}$ and $H_{2}$ and a Higgs singlet $\phi$. Although all three Higgses get VEVs, only $\mathrm{H}_{2}$ will couple to the top quark. The observed light Higgs is a linear combination of $H_{1}$ and $H_{2}$ and so could have a reduced top quark Yukawa coupling, while the Higgs with a larger coupling to the top could be much heavier. The next section details the couplings of this model and the associated constraints from the LHC searches. After electroweak symmetry breaking (EWSB), one of the Higgs mass eigenstates becomes the $125 \mathrm{GeV}$ Higgs and couples to the top with a strength proportional to the mixing angle between the neutral Higgses. FCNC can be completely avoided if the other fermions also only couple to $\mathrm{H}_{2}$, or if leptons and/or the down-type quarks couple only to $H_{1}$. As the couplings of the Higgses to fermions other than the top quark do not lead to meaningful constraints from finetuning, and can always be made consistent with the FCNC, we do not study them in detail.

Before the discovery of the Higgs boson, it was wellknown that a heavy SM Higgs boson would help to alleviate the little hierarchy problem. The authors of Refs. [26,27] used this fact to generate a heavy SM Higgs boson with a naturally raised cutoff. Such a scenario, however, required large dimensionless couplings in the scalar potential. This inevitably led to low scale Landau poles. Given this, the authors of Refs. [26,27] were able to raise the cutoff only to $\Lambda \sim 1.5 \mathrm{TeV}$ for a SM Higgs mass of about $400-600 \mathrm{GeV}$. In a one Higgs model, the only way to raise the Higgs mass is to have a large quartic coupling which leads to a Landau pole at a low scale. In multi-Higgs doublet models the possibilities are more diverse. In order to ameliorate any potential problems with large quadratic corrections from the scalar sector or low scale Landau poles, we need to limit the size of the dimensionless couplings. For two-Higgs doublet models in which we also do not allow large bare quadratic mass terms, this constraint forbids Higgs masses beyond $\mathcal{O}(100) \mathrm{GeV}$ and therefore considerably limits the extent to which the fine-tuning can be reduced. In order to allow for large Higgs masses, we introduce an additional Higgs singlet $\phi$ that interacts with the two Higgs doublets $H_{1}, H_{2}$ via a trilinear term of the form

$$
\mathcal{L}_{\phi h_{1} h_{2}} \sim A_{h} \phi H_{1} H_{2} .
$$

The coupling $A_{h}$ is now dimensionful, and its values are only constrained by perturbative unitarity and vacuum stability $[53,54]$. The contribution to the Higgs mass divergence from these couplings is at best logarithmic

$$
\delta m_{h}^{2} \sim \frac{A^{2}}{16 \pi^{2}} \log \left(\Lambda^{2} / m_{h}^{2}\right),
$$

although the coefficient of the log divergence can be large when $A$ is large.

\section{B. Scalar sector}

The fields $H_{1}$ and $H_{2}$ and the real scalar singlet $\Phi$ get VEVs $v_{1}, v_{2}$, and $u$, respectively, and have the following structure:

$$
\begin{gathered}
H_{1}=\left(\begin{array}{c}
G^{+} \cos \beta+H^{+} \sin \beta \\
\frac{1}{\sqrt{2}}\left(v_{1}+h_{1}+i\left(G^{0} \cos \beta+A^{0} \sin \beta\right)\right)
\end{array}\right), \\
H_{2}=\left(\begin{array}{c}
G^{+} \sin \beta-H^{+} \cos \beta \\
\frac{1}{\sqrt{2}}\left(v_{2}+h_{2}+i\left(G^{0} \sin \beta-A^{0} \cos \beta\right)\right)
\end{array}\right), \\
\Phi=u+\phi .
\end{gathered}
$$


Hence, in addition to the Goldstone bosons $G^{0}, G^{ \pm}$, the theory involves three neutral scalars $\left(h_{1}, h_{2}, \phi\right)$, one pseudoscalar $A^{0}$, and one charged scalar $H^{ \pm}$. As in the 2HDM, we introduce a mixing angle $\beta$ such that the VEVs of the $S U(2)$ doublets can be rewritten as

$$
v_{1}=v \cos \beta, \quad v_{2}=v \sin \beta,
$$

where $v=246 \mathrm{GeV}$ is the electroweak VEV.

As for the type-I $2 \mathrm{HDM}$, we require our model to be invariant under a $\mathbb{Z}_{2}$ symmetry. We choose for the Higgs fields to transform under this symmetry as

$$
h_{1} \rightarrow h_{1}, \quad h_{2} \rightarrow-h_{2}, \quad \Phi \rightarrow-\Phi .
$$

EWSB, however, causes this $\mathbb{Z}_{2}$ symmetry to be spontaneously broken. This is problematic cosmologically because of the formation of domain walls $[55,56]$. We assume one (or all) of the following: we break this discrete symmetry softly by small terms. Alternatively, there is a low reheating temperature after inflation [57], below the electroweak scale, so the temperature is never above the phase transition scale, and the domain walls do not form. Finally, the discrete symmetry could originate from a global $U(1)$ at higher energies. At the $U(1)$ symmetry breaking scale, cosmic strings form. Then, when the domain walls form at the electroweak scale, they end on loops of the previously formed cosmic strings and the whole stringdomain wall network is no longer stable and rapidly disappears by radiating scalars [57].

\section{Overview of the Higgs potential}

The most generic potential consistent with the $\mathbb{Z}_{2}$ symmetry discussed above and minimized around the VEVs $v_{1}, v_{2}$, and $u$ is

$$
\begin{aligned}
V= & \lambda_{1}\left(H_{1}^{\dagger} H_{1}-\frac{v_{1}^{2}}{2}-\frac{A_{h} u v_{2}}{\lambda_{1} v_{1}}\right)^{2}+\lambda_{2}\left(H_{2}^{\dagger} H_{2}-\frac{v_{2}^{2}}{2}-\frac{A_{h} u v_{1}}{\lambda_{2} v_{2}}\right)^{2}+\lambda_{3}\left(\Phi^{2}-u^{2}-\frac{A_{h} v_{1} v_{2}}{4 \lambda_{3} u}\right)^{2} \\
& +\lambda_{4}\left(H_{1}^{\dagger} H_{1}-\frac{v_{1}^{2}}{2}+H_{2}^{\dagger} H_{2}-\frac{v_{2}^{2}}{2}\right)^{2}+\lambda_{5}\left(H_{1}^{\dagger} H_{1} H_{2}^{\dagger} H_{2}-H_{1}^{\dagger} H_{2} H_{2}^{\dagger} H_{1}\right)+\lambda_{6}\left(H_{1}^{\dagger} H_{1}-\frac{v_{1}^{2}}{2}\right)\left(\Phi^{2}-u^{2}\right) \\
& +\lambda_{7}\left(H_{2}^{\dagger} H_{2}-\frac{v_{2}^{2}}{2}\right)\left(\Phi^{2}-u^{2}\right)+A_{h}\left(\Phi H_{1} H_{2}^{\dagger}+\Phi H_{1}^{\dagger} H_{2}-u v_{1} v_{2} \cos \xi\right) .
\end{aligned}
$$

The last trilinear term in Eq. (10) generates off-diagonal contributions to the scalar mass matrix of the form $A_{h} u h_{1} h_{2}$ and $A_{h} v_{1,2} h_{2,1} \phi$. Minimizing the scalar potential around the VEVs also causes this term to contribute to the diagonal elements of this scalar mass matrix as well as to the masses of the pseudoscalar $A^{0}$ and charged Higgs $H^{ \pm}$. The squared mass matrix for the neutral scalars can then be written as

$$
M_{h}^{2}=\left(\begin{array}{ccc}
2 v^{2}\left(\lambda_{1}+\lambda_{4}\right) \cos ^{2} \beta-A_{h} u \tan \beta & A_{h} u+v^{2} \lambda_{4} \sin (2 \beta) & v\left(2 u \lambda_{6} \cos \beta+A_{h} \sin \beta\right) \\
A_{h} u+v^{2} \lambda_{4} \sin (2 \beta) & -A_{h} u \cot \beta+2 v^{2}\left(\lambda_{2}+\lambda_{4}\right) \sin ^{2} \beta & v\left(A_{h} \cos \beta+2 u \lambda_{7} \sin \beta\right) \\
v\left(2 u \lambda_{6} \cos \beta+A_{h} \sin \beta\right) & v\left(A_{h} \cos \beta+2 u \lambda_{7} \sin \beta\right) & 8 u^{2} \lambda_{3}-\frac{A_{h}}{2 u} v^{2} \sin (2 \beta)
\end{array}\right) .
$$

Diagonalizing this matrix will give three scalar mass eigenstates $\left(h, h^{\prime}, h^{\prime \prime}\right)$ with masses $m_{h}, m_{h^{\prime}}$, and $m_{h^{\prime \prime}}$. As a convention for the rest of this work, we define $h, h^{\prime}$, and $h^{\prime \prime}$ as the states with the largest $h_{1}, h_{2}$, and $\phi$ components, respectively. In the limit where $\lambda_{i} \ll 1$ and $\left|A_{h}\right|, v_{1}, v_{2} \ll u$, the mass eigenstates can be approximated by

$$
\begin{aligned}
m_{1}^{2}= & 2 A_{h} v_{1}+\left(2\left(\lambda_{3} u^{2}+\lambda_{7} u v_{2}+\left(\lambda_{2}+\lambda_{4}\right) v_{2}^{2}\right)-\frac{\left(\left(2 \lambda_{3}-2 \lambda_{6}+\lambda_{7}\right) u+\left(2 \lambda_{2}-2 \lambda_{4}+\lambda_{7}\right) v_{2}\right)\left(u+v_{2}\right) v_{1}^{2}}{u v_{2}}\right) \\
& +\frac{1}{2 A_{h} v_{1} v_{2}^{2}}\left(2 \lambda_{3}\left(\lambda_{3} u^{2}-\left(\lambda_{2}+\lambda_{4}\right) v_{2}^{2}\right) u^{2} v_{2}^{2}+\left(\lambda_{3}^{2} u^{4}+\lambda_{3}\left(2 \lambda_{2}-3 \lambda_{3}-6 \lambda_{4}+4 \lambda_{6}\right) u^{2} v_{2}^{2}\right.\right. \\
& \left.\left.-\left(\lambda_{2}+\lambda_{4}\right)\left(3 \lambda_{2}-2 \lambda_{3}-5 \lambda_{4}+4 \lambda_{6}\right) v_{2}^{4}\right) v_{1}^{2}\right)+\cdots, \\
m_{2}^{2}= & -2 A_{h} v_{1}+\left(2 \lambda_{3} u^{2}-2 \lambda_{7} u v_{2}+2\left(\lambda_{2}+\lambda_{4}\right) v_{2}^{2}+\frac{\left(\left(2 \lambda_{3}-2 \lambda_{6}+\lambda_{7}\right) u-\left(2 \lambda_{2}-2 \lambda_{4}+\lambda_{7}\right) v_{2}\right)\left(u-v_{2}\right) v_{1}^{2}}{u v_{2}}\right) \\
& -\frac{1}{2 A_{h} v_{1} v_{2}^{2}}\left(2 \lambda_{3} u^{2} v_{2}^{2}\left(\lambda_{3} u^{2}-2\left(\lambda_{2}+\lambda_{4}\right) v_{2}^{2}\right)+\left(\lambda_{3}^{2} u^{4}+\lambda_{3}\left(2 \lambda_{2}-3 \lambda_{3}-6 \lambda_{4}+4 \lambda_{6}\right) u^{2} v_{2}^{2}\right.\right. \\
& \left.\left.-\left(\lambda_{2}+\lambda_{4}\right)\left(3 \lambda_{2}-2 \lambda_{3}-5 \lambda_{4}+4 \lambda_{6}\right) v_{2}^{4}\right) v_{1}^{2}\right)+\cdots,
\end{aligned}
$$




$$
\begin{aligned}
m_{3}^{2}= & 4\left(\lambda_{1}+\lambda_{2}+\lambda_{3}-\lambda_{6}+\lambda_{7}\right) v_{1}^{2} \\
& -A_{h}\left(\frac{v_{1} v_{2}}{u}+\frac{u v_{1}}{v_{2}}+\frac{u v_{2}}{v_{1}}\right)+\cdots
\end{aligned}
$$

In Appendix A, we give the neutral mass eigenstates for $\lambda_{i} \ll 1$ and $v_{1}, v_{2}, u \ll\left|A_{h}\right|$. Throughout this work, the scalar field with the largest $h_{1}$ component $h$ is taken to be the observed Higgs boson, with $m_{h}=125 \mathrm{GeV}$. In order for $h^{\prime}$ and $h^{\prime \prime}$ to be heavier than $h, A_{h}$ should be negative. Thus, the addition of the singlet $\phi$ and its associated trilinear coupling to the model helps to establish a significant mass hierarchy between the $125 \mathrm{GeV}$ Higgs and the extra scalar fields without having large quartic couplings. The mass eigenstates $\left(h, h^{\prime}, h^{\prime \prime}\right)$ are related to the interaction eigenstates $\left(h_{1}, h_{2}, \phi\right)$ by the following rotation matrix:

$$
\begin{aligned}
\left(\begin{array}{l}
h \\
h^{\prime} \\
h^{\prime \prime}
\end{array}\right)= & \left(\begin{array}{ccc}
c_{1} c_{3}-c_{2} s_{1} s_{3} & c_{3} s_{1}+c_{2} c_{1} s_{3} & s_{2} s_{3} \\
-c_{1} s_{3}-c_{2} c_{3} s_{1} & c_{1} c_{2} c_{3}-s_{1} s_{3} & c_{3} s_{2} \\
s_{1} s_{2} & -c_{1} s_{2} & c_{2}
\end{array}\right) \\
& \times\left(\begin{array}{c}
h_{1} \\
h_{2} \\
\phi
\end{array}\right),
\end{aligned}
$$

where the $c_{i}$ and $s_{i}$ represent the cosines and the sines of the Euler angles $\alpha_{i}$. We can see that the $\alpha_{2}$ angle drives the mixing of $\phi$ with the $S U(2)$ doublet states. When this mixing is small, the angle $\alpha=\alpha_{1}+\alpha_{3}$ can be interpreted as the mixing angle between $h_{1}$ and $h_{2}$. In this limit, our model becomes similar to a two-Higgs doublet model. As we will see in the rest of this section, our main difference with a standard 2HDM will be that in our scenario, the uptype quarks will couple preferentially to the beyond the standard model (BSM) Higgs bosons in the limit where $\alpha$ is low.

The masses of the charged $\left(H^{ \pm}\right)$and $C P$-odd neutral $\left(A^{0}\right)$ Higgs bosons can be obtained analytically as follows:

$$
\begin{gathered}
m_{H^{ \pm}}^{2}=\frac{\lambda_{5}}{2} v^{2}-\frac{A_{h} u}{\cos \beta \sin \beta}, \\
m_{A^{0}}^{2}=-\frac{A_{h} u}{\cos \beta \sin \beta} .
\end{gathered}
$$

Here, the role of the trilinear term $A_{h} h_{1} h_{2} \phi$ in generating a mass hierarchy between the SM Higgs $h$ and the BSM Higgses is obvious as this term leads to the $\left|A_{h}\right| u$ contributions in Eq. (17).

\section{Vacuum stability and perturbativity}

In order for the Higgs potential shown in Eq. (10) to be valid, it needs to be bounded from below, and the quartic and trilinear couplings need to satisfy perturbativity and unitarity requirements. In order for the potential to not go to minus infinity for large values of the scalar fields, we require the quartic couplings to satisfy the following conditions, taken from [58]:

$$
\begin{gathered}
\lambda_{1,2}+\lambda_{4}>0, \quad\left|\lambda_{6}\right|<4 \sqrt{\lambda_{3}\left(\lambda_{1}+\lambda_{4}\right)}, \\
\lambda_{3}>0, \quad\left|\lambda_{7}\right|<4 \sqrt{\lambda_{3}\left(\lambda_{2}+\lambda_{4}\right)}, \\
\lambda_{4}+\lambda_{5}>-\sqrt{\left(\lambda_{1}+\lambda_{4}\right)\left(\lambda_{2}+\lambda_{4}\right)}, \\
\lambda_{4}+\lambda_{5} / 2>-\sqrt{\left(\lambda_{1}+\lambda_{4}\right)\left(\lambda_{2}+\lambda_{4}\right)} .
\end{gathered}
$$

In addition for $\lambda_{6}<0$ or $\lambda_{7}<0$, we also require

$$
\begin{aligned}
& -\frac{1}{2} \lambda_{6} \lambda_{7}+4 \lambda_{3}\left(2 \lambda_{4}+\lambda_{5}\right) \\
& >-\sqrt{4\left(4 \lambda_{3}\left(\lambda_{1}+\lambda_{4}\right)-\lambda_{6}^{2} / 4\right)\left(4 \lambda_{3}\left(\lambda_{2}+\lambda_{4}\right)-\lambda_{7}^{2} / 4\right)}, \\
& -\frac{1}{2} \lambda_{6} \lambda_{7}+8 \lambda_{3}\left(\lambda_{4}+\lambda_{5}\right) \\
& >-\sqrt{4\left(4 \lambda_{3}\left(\lambda_{1}+\lambda_{4}\right)-\lambda_{6}^{2} / 4\right)\left(4 \lambda_{3}\left(\lambda_{2}+\lambda_{4}\right)-\lambda_{7}^{2} / 4\right)} .
\end{aligned}
$$

In addition to the above requirements, the quartic couplings $\lambda_{i}$ also need to remain perturbative up to at least the cutoff scale $\Lambda$ at which new physics should appear. In the rest of this work, for each scale $\Lambda$ that we consider, we require the scalar quartic couplings to satisfy $\left|\lambda_{i}\right|<4 \pi$ and still fulfill the vacuum stability conditions at the cutoff. These couplings are large but still in the regime where perturbation theory is not unreasonable, with Landau poles still somewhat above the cutoff scale. Our renormalization group equations (RGEs) for $\lambda_{i}, A_{h}$, and the top quark Yukawa coupling are shown in Appendix C. Besides an extended Higgs sector, our model will have modified couplings of the $125 \mathrm{GeV}$ Higgs to fermions and gauge bosons. While the couplings of the Higgs to gauge bosons have been measured to be within $10 \%$ of the SM ones, the measurements of the Higgs couplings to fermions either have been indirect or have large uncertainties. In the rest of this section, we discuss the impact of the Higgs-gauge coupling measurements on the mixing angles of the neutral scalars and how the large uncertainty on the Higgs to top coupling measurement can be exploited to alleviate the fine-tuning problem.

\section{Yukawa couplings to fermions}

In standard two-Higgs doublet models, the up-type quarks couple preferentially to the interaction eigenstate that contributes mostly to the SM-like Higgs $h$ in the low mixing limit. In our model, in order to obtained a suppressed coupling of the $125 \mathrm{GeV}$ Higgs to the top quark, we 
assume that $h$ is mostly $H_{1}$ while the up-type quarks couple only to $H_{2}$. These constraints can be the result of a $\mathbb{Z}_{2}$ symmetry under which the right-handed up-type quarks such as $t_{R}$ transform as

$$
t_{R} \rightarrow-t_{R}
$$

and $H_{2} \rightarrow-H_{2}$. In order to avoid problematic FCNCs, we may take the down-type quarks and leptons to couple only to either $H_{1}$ or $H_{2}$. The couplings of the Higgs bosons to the quarks can then be written as

$$
\mathcal{L}_{t}=\lambda_{u} q_{L} u_{R} h_{2}+\lambda_{d} q_{L} d_{R} h_{1}
$$

The couplings of the Higgses to leptons will not be relevant for this study. In the rest of this work, we will focus particularly on the couplings of the Higgses to the top and the bottom quarks that can be written as

$$
\mathcal{L}_{t}=\lambda q_{L} t_{R} h_{2}+\lambda_{b} q_{L} b_{R} h_{1} .
$$

In order to obtain the correct top and bottom quark masses $m_{t}$ and $m_{b}$, the strength of the couplings $\lambda$ and $\lambda_{b}$ should be the following:

$$
\lambda=\frac{\lambda_{t}^{\mathrm{SM}}}{\sin \beta}, \quad \lambda_{b}=\frac{\lambda_{b}^{\mathrm{SM}}}{\cos \beta} .
$$

The coupling of the top quark to $h^{\prime}$ is thus typically larger than 1, which could lead to low scale Landau poles. Requiring no Landau poles for $\lambda_{t}^{\prime}$ up to a given cutoff scale $\Lambda$ leads to an upper bound on the value of $\lambda_{t}^{\prime}$ at the electroweak (EW) scale that translates in turn into a lower bound on $\beta$. This lower bound is shown as a function of $\Lambda$ in Fig. 1. The RGEs for the top quark and strong couplings that we solved to obtain this result are shown in Appendix C. Conversely, the LHC measurements of the $125 \mathrm{GeV}$ Higgs coupling to $b \bar{b}$ as well as perturbativity requirements on $\lambda_{b}$ should provide an upper bound on $\beta$. Since the uncertainties on the Higgs coupling to $b$-quark measurements at the LHC

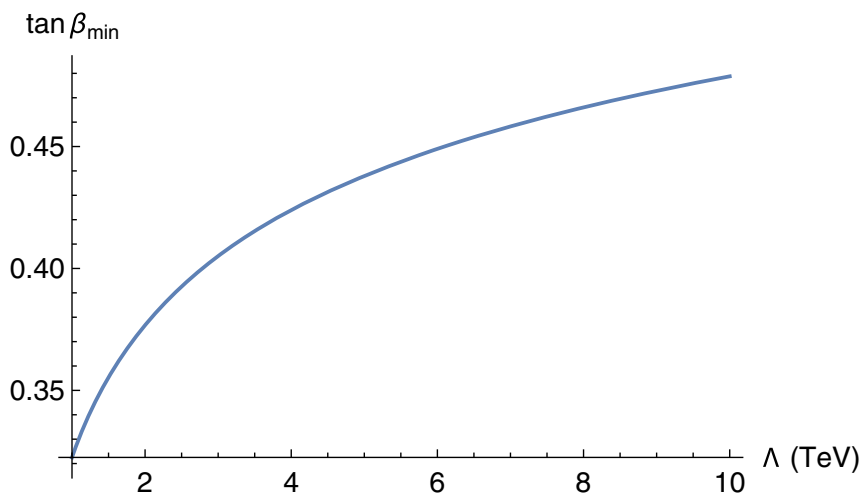

FIG. 1. Minimum value of $\tan \beta$ allowed by requiring no Landau poles below $\Lambda$ for the top quark coupling to $h^{\prime}$. are huge and $\lambda_{b}^{\mathrm{SM}}$ is very small, however, this upper bound is expected to be extremely loose and we do not take it into account in the rest of this work.

When the mixing angles between the scalars are small, the top quark should couple preferentially to $h^{\prime}$ - the mass eigenstate most similar to $h_{2}$-while the top coupling to $h$, $\lambda_{t}$, will be mixing suppressed. This coupling can be written as a function of the mixing angles in Eq. (15) to obtain

$$
\lambda_{t}=\lambda_{t}^{\mathrm{SM}} \frac{c_{3} s_{1}+c_{2} c_{1} s_{3}}{s_{\beta}} .
$$

In the limit where the $\phi$ mixing to $h_{1}$ and $h_{2}$ is small, this coupling can be approximated by

$$
\lambda_{t} \approx \lambda_{t}^{\mathrm{SM}} \frac{\sin \alpha}{\sin \beta},
$$

where $\alpha=\alpha_{1}+\alpha_{3}$ is the mixing angle between $h_{1}$ and $h_{2}$. In order for this coupling to be significantly lower than the SM coupling, it is therefore crucial to depart from the alignment limit, with $\alpha<\beta$. Going away from the alignment limit could, however, significantly modify the couplings of the $125 \mathrm{GeV}$ Higgs to other SM particles. In most cases, these deviations are not directly highly constrained at the LHC since the Higgs couplings to the other SM fermions have not been precisely measured to date. The Higgs couplings to photons and gluons do indirectly constrain the fermion couplings more precisely; however, these can also be easily modified by introducing higher dimensional operators or other new physics so we do not necessarily need to consider these indirect constraints on the fermion couplings. The Higgs couplings to vector bosons, on the other hand, are tree level and have been measured to a fairly good level of precision at the LHC. It is therefore crucial to determine how a modification of $\lambda_{t}$ would affect these couplings in our model.

\section{Gauge sector}

The electroweak gauge bosons couple to the Higgs doublets through the standard covariant derivative

$$
D \mathcal{H}=\partial \mathcal{H}-i g_{2} W \mathcal{H}-i g_{1} Y \mathcal{H} .
$$

Here $\mathcal{H}=\left(h_{1}, h_{2}\right)$, and $g_{2}$ and $g_{1}$ are the $S U(2)_{L}$ and $U(1)_{Y}$ gauge couplings. Using the definitions introduced in Eq. (7) for the Higgs fields, we can readily check that we obtain the correct masses for the gauge bosons. Using the notation defined in Eq. (15) for the mixing angles, the light Higgs coupling to the gauge bosons reads as

$$
g_{h V V}=g_{h V V}^{\mathrm{SM}}\left[c_{3} \cos \left(\beta-\alpha_{1}\right)+c_{2} s_{3} \sin \left(\beta-\alpha_{1}\right)\right],
$$

which is always smaller than one. In order to be consistent with the current LHC results [20], $g_{h V V}$ needs to be of at 
least $90 \%$ of the SM value. In order to understand the implications of these constraints, we consider first the case where $c_{2} \approx 1$, which corresponds to a scenario where $\phi$ mixes very little with the scalar components of the Higgs doublets. In this limit, the coupling between $h$ and the gauge bosons becomes

$$
g_{h V V}=g_{h V V}^{\mathrm{SM}} \cos (\beta-\alpha),
$$

where again $\alpha=\alpha_{1}+\alpha_{3}$. In order for this coupling to be close to the SM value, we therefore need to be in the alignment limit where $\alpha$ and $\beta$ are close to each other. This requirement might be in tension with our end goal of reducing the top coupling to $h$ defined in Eq. (27), the latter being close to one in this limit. The current LHC results, however, still leave some significant freedom since the requirement that $g_{h V V} \geq 0.9 g_{h V V}^{\mathrm{SM}}$ translates into

$$
|\beta-\alpha| \leq 0.45 \text {. }
$$

The values of $\lambda_{t}$ and $\lambda_{t}^{\prime}$ for $\alpha=\beta-0.45$ are shown as a function of $\beta$ in Fig. 2. As can be inferred from both this figure and Fig. 1, it is a priori possible to considerably reduce the value of the top coupling to the $125 \mathrm{GeV}$ Higgs without significantly reducing its coupling to gauge bosons or introducing Landau poles below at least $5 \mathrm{TeV}$ from the running of $\lambda_{t}^{\prime}$. Note that the top Yukawa coupling is most suppressed for low values of $\beta$, so the coupling of the $125 \mathrm{GeV}$ Higgs to bottom quarks is SM-like and well within the LHC limits.

When the singlet $\phi$ mixes with $h_{1}$ and $h_{2}$, the reasoning outlined above still applies. Since the mixing between the

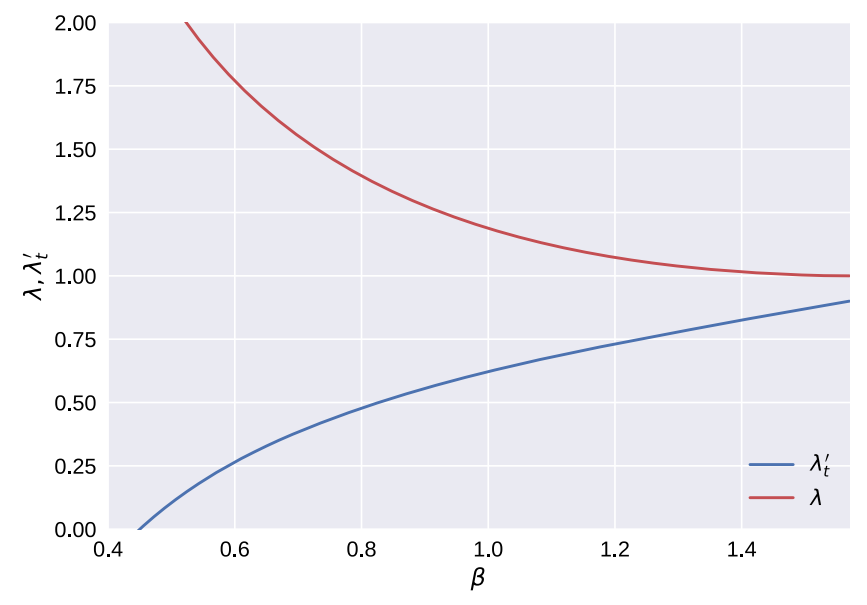

FIG. 2. Value of $\lambda_{t}^{\prime}$ when the mixing angle $\alpha$ between the two Higgs doublets is set to $\alpha=\beta-0.45$, that is, the minimum value allowed by the Higgs-gauge coupling measurements (blue line). The red line shows the top coupling to $H_{2} \lambda$, defined in Eq. (25). We can see that, if the mixing angle $\alpha$ is free to vary as far from $\beta$ as allowed by the LHC measurements, it is possible to get an extremely suppressed top coupling to the $125 \mathrm{GeV}$ Higgs while still having $\lambda$ remain perturbative up to at least $5 \mathrm{TeV}$. scalar singlet and the scalar components of the doublets is governed by $\alpha_{2}$, increasing this mixing would translate into decreasing $c_{2}$. In order for the $g_{h V V}$ coupling in Eq. (31) to remain close to the SM value, $c_{3} \cos \left(\beta-\alpha_{1}\right)$ needs to increase. These constraints push us into a region of parameter space where $\alpha_{3}$ is small and the mixing between $h_{1}$ and $h_{2}$ is governed by $\alpha_{1}$. This scenario is qualitatively similar to the one where the singlet $\phi$ is decoupled and the steps detailed above can be repeated with $\alpha_{1}$ substituted to $\alpha$.

As our results show, strongly reducing the $125 \mathrm{GeV}$ Higgs coupling to the top quark without creating tension with the current LHC results or introducing low scale Landau poles can be achieved for certain values of the mixing angles between the neutral scalars. It is crucial in particular that the effective mixing angle between $h_{1}$ and $h_{2}$ is as far from $\beta$ as allowed by the current Higgs-gauge coupling measurements. This requirement, however, constrains the product $\sqrt{\left|A_{h}\right| u}$, appearing in the mass matrix (11) to not be too large compared to the EW scale. Consequently, the regions of parameter space where the $125 \mathrm{GeV}$ Higgs coupling to the top quarks is the lowest are also regions where the other Higgses have $\mathcal{O}(100) \mathrm{GeV}$ masses. These other Higgses, however, can exhibit large quadratic divergences that could be reduced only by reintroducing sizable fine-tuning. In particular, by construction, $h^{\prime}, A^{0}$, and $H^{ \pm}$are associated with order one top Yukawa couplings and therefore need to be much heavier than $h$. In what follows, we discuss how we impose the naturalness requirement and compute the dominant fine-tuning factors.

\section{NATURALNESS}

At one-loop the quadratic divergences generated by the minimal model for the masses of any of the Higgses can be represented as

$$
\delta m_{h_{i}}^{2}=\alpha_{t} \Lambda_{t}^{2}+\alpha_{g} \Lambda_{g}^{2}+\alpha_{h} \Lambda_{h}^{2},
$$

where we have followed the notation in [26]. In what follows, we will neglect the contributions from the gauge boson loops $\alpha_{g}$ due to the low values of the weak couplings. The quadratic divergences from the top and Higgs boson loops in our model can then be derived from the ColemanWeinberg potential

$$
\begin{aligned}
V_{\text {quadratic }}= & \frac{\Lambda^{2}}{32 \pi^{2}}\left(\lambda_{1}+2 \lambda_{4}+\frac{1}{2} \lambda_{5}+\frac{1}{2} \lambda_{6}\right) H_{1}^{\dagger} H_{1} \\
& +\frac{\Lambda^{2}}{32 \pi^{2}}\left(\lambda_{2}+2 \lambda_{4}+\frac{1}{2} \lambda_{5}+\frac{1}{2} \lambda_{7}\right) H_{2}^{\dagger} H_{2} \\
& +\frac{\Lambda^{2}}{32 \pi^{2}}\left(\lambda_{3}+\frac{\lambda_{6}}{2}+\frac{\lambda_{7}}{2}\right) \Phi^{2}-\frac{3 \lambda^{2} \Lambda^{2}}{8 \pi^{2}} H_{2}^{\dagger} H_{2} .
\end{aligned}
$$


We discuss our derivation in more detail in Appendix B. The values of the fine-tuning factors $\alpha_{i}$ can be deduced by rotating into the mass basis and computing the derivatives of $V_{\text {quadratic }}$ with respect to the different fields. For the light Higgs $h$, we obtain

$$
\begin{aligned}
\alpha_{t h}= & \frac{3 \lambda_{t}^{2}}{4 \pi^{2}}, \\
\alpha_{h h}= & \alpha_{h 11}\left(c_{1} c_{3}-c_{2} s_{1} s_{3}\right)^{2}+\alpha_{h 22}\left(s_{1} c_{3}+c_{2} c_{1} s_{3}\right)^{2} \\
& +\alpha_{h 33}\left(s_{2} s_{3}\right)^{2},
\end{aligned}
$$

where $\lambda_{t}$ is defined in Eq. (27) and the $\alpha_{h i i}$ are defined by

$$
\begin{gathered}
\alpha_{h 11}=-\frac{\left(2 \lambda_{1}+4 \lambda_{4}+\lambda_{5}+\lambda_{6}\right)}{16 \pi^{2}}, \\
\alpha_{h 22}=-\frac{\left(2 \lambda_{2}+4 \lambda_{4}+\lambda_{5}+\lambda_{7}\right)}{16 \pi^{2}}, \\
\alpha_{h 33}=-\frac{\left(2 \lambda_{3}+\lambda_{6}+\lambda_{7}\right)}{16 \pi^{2}}
\end{gathered}
$$

and are weighted by combinations of the cosines and sines of the mixing angles $\alpha_{i}$, defined in Eq. (15). The coupling $\lambda_{t}$ and the Higgs quartic couplings are evaluated at the cutoff scale $\Lambda$. Usually, when the quartic couplings $\lambda_{i}$ are taken to be perturbative, these quadratic divergences lead to a lower fine-tuning than the ones from the top loops. Similarly, we derive the fine-tuning factors for the other two Higgses:

$$
\begin{aligned}
\alpha_{t h^{\prime}}= & \frac{3 \lambda_{t}^{\prime 2}}{4 \pi^{2}}, \\
\alpha_{h h^{\prime}}= & \alpha_{h 11}\left(-c_{1} s_{3}-c_{2} s_{1} c_{3}\right)^{2}+\alpha_{h 22}\left(c_{1} c_{2} c_{3}-s_{1} s_{3}\right)^{2} \\
& +\alpha_{h 33}\left(s_{2} c_{3}\right)^{2}, \\
\alpha_{t h^{\prime \prime}}= & \frac{3 \lambda^{2}\left(c_{1} s_{2}\right)^{2}}{4 \pi^{2}}, \\
\alpha_{h h^{\prime \prime}}= & \alpha_{h 11}\left(s_{1} s_{2}\right)^{2}+\alpha_{h 22}\left(c_{1} s_{2}\right)^{2}+\alpha_{h 33} c_{2}^{2} .
\end{aligned}
$$

Finally, the pseudoscalar and charged Higgses are also associated with large quadratic divergences. The corresponding fine-tuning factors are

$$
\begin{aligned}
\alpha_{t\left\{A, H^{ \pm}\right\}} & =\frac{3 \lambda^{2} \cos ^{2} \beta}{4 \pi^{2}}, \\
\alpha_{h\left\{A, H^{ \pm}\right\}} & =\alpha_{h 11} \sin ^{2} \beta+\alpha_{h 22} \cos ^{2} \beta .
\end{aligned}
$$

The sensitivity of the SM Higgs masses to a given cutoff scale $\Lambda_{i}$ is given by the formula

$$
D\left(m_{h}\right)=\left|\frac{\partial \log m_{h}^{2}}{\partial \log \Lambda_{i}^{2}}\right|=\frac{\left|\alpha_{i}\right| \Lambda_{i}^{2}}{m_{h}^{2}} .
$$

In the rest of this work, the fine-tuning factor that we consider at a given scale will be the maximal value of the fine-tunings associated with the top, gauge boson, or Higgs loops, for all three Higgs bosons

$$
D_{\max }(\Lambda)=\max _{j=\left\{h, h^{\prime}, h^{\prime \prime}, A, H^{ \pm}\right\}}\left\{\frac{\left|\alpha_{t j}+\alpha_{h j}\right| \Lambda^{2}}{m_{j}^{2}}\right\} .
$$

This estimate is conservative since it assumes that the cutoff scales for all the loop contributions to the Higgs masses will be at their lowest possible values for a given $D_{\max }$. Since $\alpha_{t j}$ and $\alpha_{h j}$ are of opposite signs, they are expected to cancel out, either partially or, if the Veltman condition 1 is fulfilled, totally.

Besides looking for parameters with low total finetuning, we also must consider the current LHC searches for new bosons. In the next section, we detail what searches and decay channels are relevant to our models and how we implement the corresponding constraints.

\section{LHC PHENOMENOLOGY}

As highlighted in Sec. II D, in order to be as far as possible from the alignment limit, it is necessary for either $h^{\prime}$ or $h^{\prime \prime}$ to be light, with masses typically below a TeV. If $h^{\prime}$ is light, in particular, the model will also involve a light pseudoscalar $A^{0}$ and charged Higgs $H^{ \pm}$, which could both be within the reach of the corresponding LHC searches. At low $\tan \beta$ in the minimal supersymmetric standard model (MSSM), which is also the preferred region for our low fine-tuning models as discussed in Sec. II C, pseudoscalar Higgses are excluded up to about $400 \mathrm{GeV}$. It is therefore crucial to investigate how the different Higgs searches at $13 \mathrm{TeV}$ LHC will constrain our models.

As discussed in detail in Sec. II D, the couplings of the $125 \mathrm{GeV}$ Higgs to the gauge bosons are constrained to be SM-like, and the couplings to the bottom quark are expected to be much smaller than one. The couplings of these particles to the new Higgs bosons will therefore be suppressed, and the corresponding LHC searches should not be particularly sensitive to our model. Similarly, the top quark couplings to $h^{\prime \prime}$ are expected to be mixing suppressed, which would lead to reduced gluon fusion production rates. The second Higgs $h^{\prime}$, as well as $A^{0}$ and $H^{ \pm}$, however, have the following couplings to the top quarks

$$
\lambda_{t}^{\prime} \approx \frac{\lambda_{t}^{\mathrm{SM}}}{\sin \beta}, \quad \lambda_{t}^{A^{0}, H^{ \pm}}=\frac{\lambda_{t}^{\mathrm{SM}}}{\tan \beta} .
$$

When $\tan \beta$ is of order one or lower, these couplings will be of the same order as, if not larger than, the SM top Yukawa couplings. These new Higgses will therefore have sizable production rates through gluon fusion at the LHC and should therefore be severely constrained by the current searches. 
In MSSM models with $\tan \beta \lesssim 3$, heavy pseudoscalar and charged Higgs bosons have already been excluded up to about $350 \mathrm{GeV}$ [59-63]. In order to account for the possible mild suppressions of the production rates of these particles in our model, we focus on parameter points where $h^{\prime}, A^{0}$, and $H^{ \pm}$all have masses larger than $250 \mathrm{GeV}$, which correspond to the lowest masses explored by the $13 \mathrm{TeV}$ LHC Higgs searches. For these masses, the main decay modes are

$$
\begin{gathered}
h^{\prime}, h^{\prime \prime} \rightarrow t \bar{t}, b \bar{b}, Z Z, h h, W^{+} W^{-}, \\
A^{0} \rightarrow Z h, t \bar{t}, b \bar{b}, \\
H^{ \pm} \rightarrow t b, W^{ \pm} h,
\end{gathered}
$$

and the main production modes are

$$
\begin{gathered}
g g, V V \rightarrow h^{\prime}, h^{\prime \prime}, \\
g g \rightarrow A^{0}, \\
b \bar{b} \rightarrow A^{0}, \\
g \bar{b} \rightarrow t H^{+} .
\end{gathered}
$$

The $13 \mathrm{TeV}$ LHC searches for heavy BSM Higgs bosons are [59-66] and target all the decay channels shown in (46) except $h^{\prime} / A \rightarrow t \bar{t}$ and $H^{ \pm} \rightarrow W^{ \pm} h$, which is expected to be largely subdominant to $H^{ \pm} \rightarrow t b$. In what follows, for each parameter point of our model, we compute the branching ratios corresponding to the decay modes shown in (46) using the formulas given in [67]. We take the production cross sections from the LHC Higgs cross section working group and rescale them by the following $\kappa$ factors:

$$
\begin{gathered}
\kappa_{g g h^{\prime}}=\frac{\left(c_{1} c_{2} c_{3}-s_{1} s_{3}\right)^{2}}{\tan ^{2} \beta}, \\
\kappa_{g g A^{0}}=\frac{1}{\tan ^{2} \beta}, \\
\kappa_{b b A^{0}}=\tan ^{2} \beta, \\
\kappa_{V V h^{\prime}}=\left[-s_{3} \cos \left(\beta-\alpha_{1}\right)+c_{2} c_{3} \sin \left(\beta-\alpha_{1}\right)\right]^{2}, \\
\kappa_{g b H^{ \pm}}=\left[-\frac{1}{\tan \beta}+\frac{m_{b}}{m_{t}} \tan \beta\right]^{2},
\end{gathered}
$$

where the mixing parameters $\alpha_{1}, c_{123}$, and $s_{123}$ are defined in (15). For each channel, we finally compare the values of $\sigma \times \mathrm{Br}$ to the corresponding LHC limits.

\section{PARAMETER SPACE AND RESULTS}

We now scan over the parameter space of our multiple Higgs model to determine how much the fine-tuning can be lowered without breaking perturbativity or being at odds with the LHC results. Our model involves ten parameters: seven quartic couplings $\lambda_{i}$, the mixing angle $\beta$ between the VEVs of the Higgs doublets, the trilinear coupling $A_{h}$, and the VEV $u$ of the singlet $\Phi$. After requiring $m_{h}=125 \mathrm{GeV}$, the parameter space is then nine-dimensional. Such a large parameter space is particularly difficult to explore. We would therefore like to stress that our final result will be conservative, as narrow regions with low fine-tuning might have been overlooked.

In what follows, we choose a cutoff scale $\Lambda=5 \mathrm{TeV}$ and perform a uniform random scan over the following parameters:

$$
\begin{gathered}
u \in[0,5] \mathrm{TeV}, \\
\lambda_{i}^{\prime} \in[-2,2], \\
\beta \in\left[0, \frac{\pi}{2}\right],
\end{gathered}
$$

and fix $A_{h}$ by setting the lightest Higgs mass to be $125 \mathrm{GeV}$. We emphasize choosing a common cutoff scale $\Lambda$ is very conservative. The gauge boson, scalar, and top sectors could have different cutoffs which would allow a much larger number of models that meet our scan criterion. The $\lambda_{i}^{\prime}$ couplings are linear combinations of the $\lambda_{i}$ couplings and are defined in Appendix C. These combinations are the ones that enter the RGEs and are therefore more relevant to scan over from a perturbativity point of view. We scan over $10^{9}$ points and select the models verifying the vacuum stability and perturbativity constraints discussed in Sec. II and for which the couplings of the $125 \mathrm{GeV}$ Higgs to the gauge bosons are within $10 \%$ of the corresponding SM values. Additionally, in order to ensure that our results will not be influenced by the physics at the cutoff scale we consider only models where the new particles have masses below $1 \mathrm{TeV}$. Finally, we select all points for which the finetuning factor $D$ is less than 100 . These points will be represented in blue in the figures shown in this section. For each of these points and for the different BSM Higgs bosons, we compute the cross-section times branching ratio for each of the production and decay channels listed in Sec. IV and compare it to the results from the ATLAS searches [59-61,64-66]. We consider that a parameter point is not excluded at the LHC if $h^{\prime}, A^{0}$, and $H^{ \pm}$are all heavier than $250 \mathrm{GeV}$ and, for each detection channel, the ratio of the $\sigma \times \mathrm{Br}$ over the $95 \%$ confidence limit found by ATLAS is less than 1. In order to account for the important fluctuations of the ATLAS exclusion bounds at low Higgs masses as well as estimate the reach of the future 


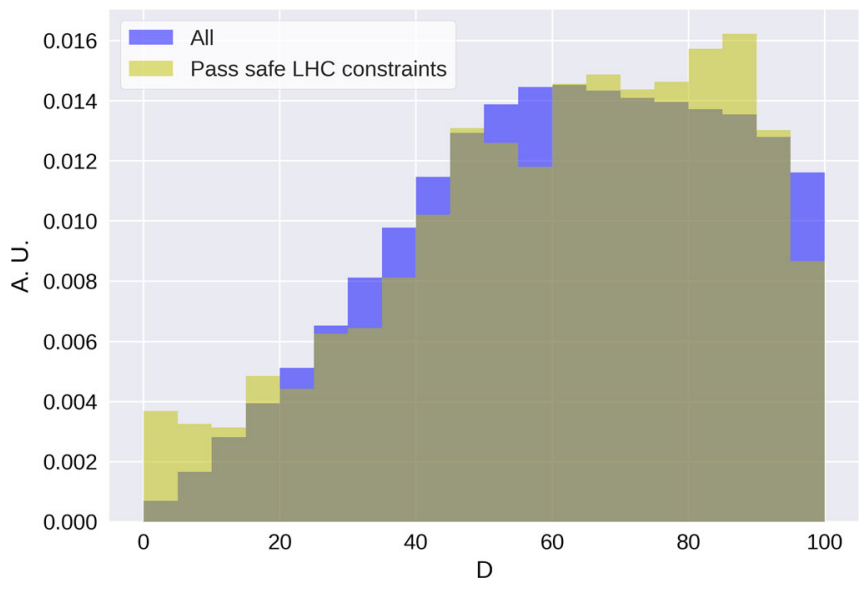

FIG. 3. Fine-tuning factor $D$ for all the points with $g_{V}>0.9$ and $D<100$ (blue) and for the subset of these points that satisfy the safe LHC constraints defined in the main text (yellow). The vertical axis is in arbitrary units with different scales for the blue and for the yellow.

LHC searches, we also define a "safe" region where the ratio of the $\sigma \times \mathrm{Br}$ over the 95\% ATLAS confidence limit for each detection channel is less than 0.1 .

Figure 3 shows the normalized distributions of the fine-tuning factors $D$ for all the points with $g_{V}>0.9$ and $D<100$ (blue) as well as the subset of these points that satisfy the LHC constraints defined above (yellow). Whether the LHC constraints are taken into account or not, the fine-tuning factor $D$ can easily reach values smaller than 10 or even 1 . Thus, lowering the top quark coupling while in the same time exploiting the partial cancellation of the top and scalar one-loop contributions to the Higgs mass could potentially be a way to reduce the fine-tuning in the SM without introducing too much complexity.

In order to understand the interplay between the suppression of the top Yukawa coupling and the cancellation from scalar couplings, we show the fine-tuning factor $D$ as a function of $\lambda_{t}$ in Fig. 4 . When the LHC constraints are not introduced, this figure shows two distinct low fine-tuning regions: one region where the top Yukawa coupling is reduced to values as low as 0.55 for $D<20$, and one where the top coupling to the SM Higgs is unsuppressed and the reduction of the fine-tuning is entirely due to the other scalars. The latter region involves new Higgses that are typically heavy due to a large quartic coupling, and therefore outside the reach of the LHC. Conversely, most of the first region has already been probed by the LHC. This result is due to the fact that such low values of the finetuning require the top coupling of the $125 \mathrm{GeV}$ Higgs $\lambda_{t}$ to be significantly reduced. In Sec. II D, we already argued that $\tan \beta$ cannot be too small in order for the top Yukawa coupling of the $h_{2}$ to remain perturbative, which in turn requires models with suppressed $\lambda_{t}$ to be far from the alignment limit. This numerical study then shows that these two conflicting constraints prevent models with reduced $\lambda_{t}$



FIG. 4. Fine-tuning $D$ versus $\lambda_{t}$ for all the points with $g_{V}>0.9$ and $D<100$ (blue dots), the points that satisfy both $D<20$ and the LHC constraints (red triangles), and the subset of these points that satisfy the "safe" LHC constraints (yellow stars). Both LHC constraints are defined in Sec. V.

to have BSM Higgses heavier than a few hundreds of GeV. In fact, our results show that the only way to obtain suppressed top Yukawa couplings is to have large mixings between the scalars, which can happen only in the low mass regime. Although a few points with $D \lesssim 10$ still survive the current constraints, especially for $\lambda_{t} \gtrsim 0.7$, they are expected to be probed by the next LHC runs. The hypothesis that the fine-tuning of the $125 \mathrm{GeV}$ Higgs mass is reduced by suppressing the top quark Yukawa coupling should therefore be fully tested in the near future.

Figures 5 and 6 show the mass scales corresponding to the regions of the parameter space with the lowest finetuning, in the $\left(u, A_{h}\right)$ plane and in the $\left(m_{h^{\prime}}, m_{h^{\prime \prime}}\right)$ plane. Although there are narrow regions with either $u \ll\left|A_{h}\right|$ or

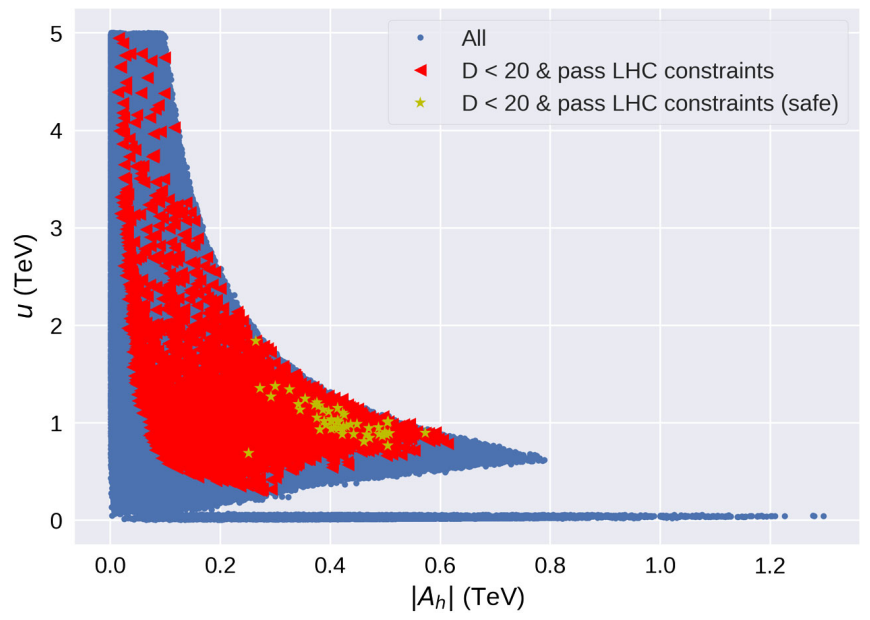

FIG. 5. $u$ versus $A_{h}$ for all the points with $g_{V}>0.9$ and $D<$ 100 (blue dots), the points that satisfy both $D<20$ and the LHC constraints (red triangles), and the subset of these points that satisfy the "safe" LHC constraints (yellow stars). 


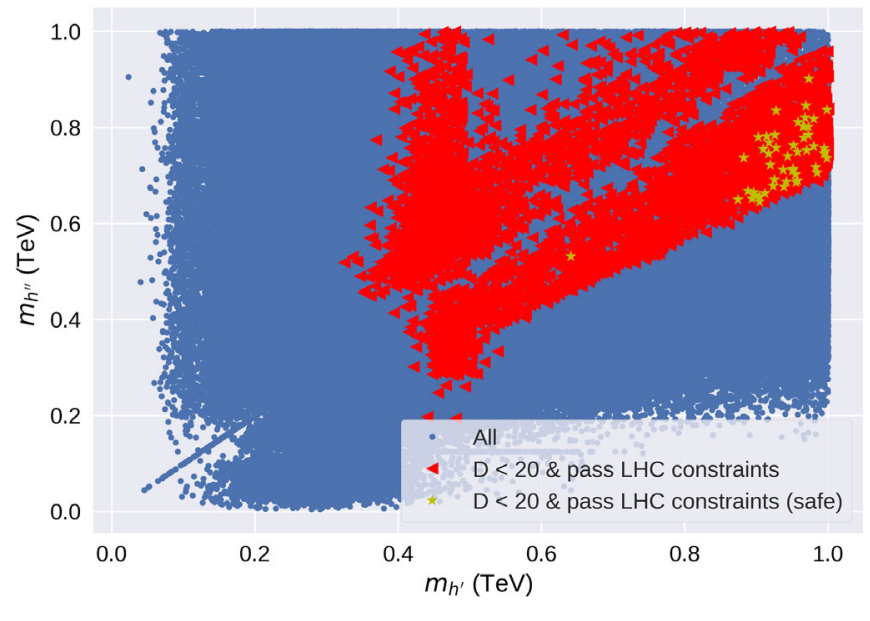

FIG. 6. $m_{h^{\prime \prime}}$ versus $m_{h^{\prime}}$ for all the points with $g_{V}>0.9$ and $D<100$ (blue dots), the points that satisfy the LHC constraints (red triangles), and the subset of these points that pass the "safe" LHC constraints (yellow stars).

$m_{h^{\prime \prime}} \gg m_{h^{\prime}}$, the low fine-tuning points that pass the safe LHC constraints generally have $\left|A_{h}\right|$ and $u$ being of the same order of magnitude. In most of the parameter space verifying these constraints, we also note that $h^{\prime \prime}$ is lighter than $h^{\prime}$. Although most of the fully degenerate limit is already excluded, the points that survive the current LHC constraints generally have $m_{h^{\prime}} \sim m_{h^{\prime \prime}}$.

Finally, Fig. 7 shows the maximal value of the $\lambda_{i}^{\prime}$ couplings defined in Appendix $\mathrm{C}$ at the cutoff scale $\Lambda=5 \mathrm{TeV}$ as a function of the fine-tuning $D$. Although, in principle, these couplings could reach values down to 2 for $D<20$, once the LHC constraints are introduced, these couplings have to be larger than 4. This result is due to the fact that these LHC constraints disfavor the regions of parameter space with low top Yukawa $\lambda_{t}$ and large quartic couplings are therefore required in order to cancel the unsuppressed top loop

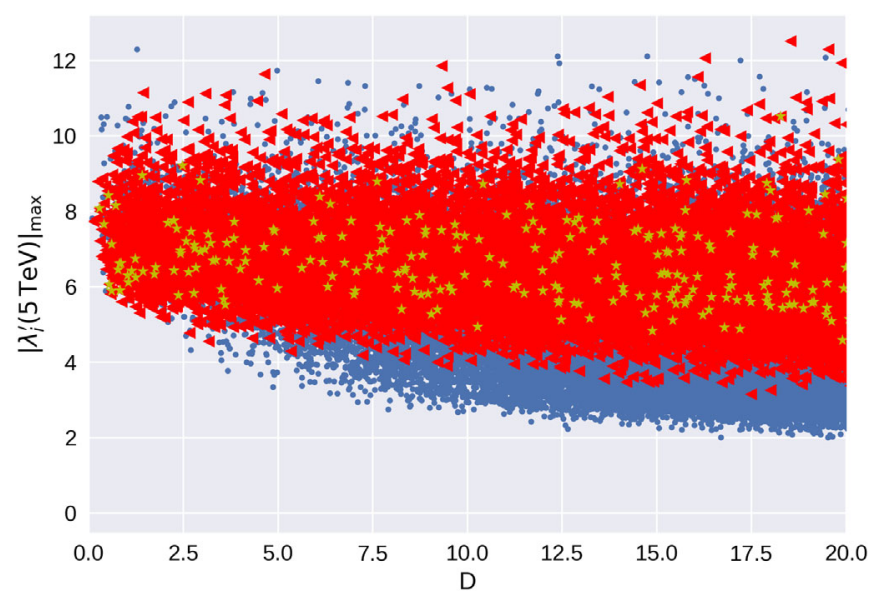

FIG. 7. Maximal value of the couplings $\lambda_{i}^{\prime}$ defined in Appendix C at $5 \mathrm{TeV},\left|\lambda_{i}^{\prime}(5 \mathrm{TeV})\right|_{\max }$, versus fine-tuning for all the points with $g_{V}>0.9$ and $D<100$ (blue dots), the points that satisfy the LHC constraints (red triangles), and the subset of these points that pass the "safe" LHC constraints (yellow stars). contribution to the Higgs mass, as shown in Eq. (1). Reducing the total fine-tuning in our model therefore suggests a strongly coupled Higgs sector at a few $\mathrm{TeV}$.

\section{BEYOND}

We have discussed how the Higgs mass parameter is calculable from the bare mass and radiative corrections, as shown in Eq. (1). In Sec. I A we have posed the question of whether the bare mass and the one-loop radiative corrections need to be fine-tuned. This is not possible in the minimal standard model unless the cutoff scale is below a $\mathrm{TeV}$ - a scale the LHC has substantially explored. In the previous sections, we focused on a minimal model that illustrates aspects of the first and third Veltman conditions in Sec. I A. We now briefly discuss the second condition. We briefly note that theories with softly broken shift symmetries can predict light scalars [68,69]. Extensions of this idea developed into little Higgs model building $[7,8,69]$. Here top partners were introduced to cancel the quadratic sensitivity to the cutoff scale from radiative corrections due to the top coupling. Given the second Veltman condition, it is conceivable that the cancellations just occur between the top and additional scalars for no obvious symmetry reason, or that the observed scalar does not have such a large top coupling as to require top partners. We consider this possibility in future work.

We also note that there is lattice gauge theory evidence that some strongly coupled theories have scalars which are much lighter than the strong coupling scale for a dynamical reason which is not related to a symmetry [70].

\section{CONCLUSIONS}

We have considered the cutoff sensitivity of an extension of the minimal standard model with additional particles which only carry electroweak charges, and we argue that they should be scalars. Our goals are more modest that those of theorists who achieve cancellations in the Higgs mass from symmetry considerations, but we do not have to pay the price of introducing new colored top partners or a new strong group, which therefore makes it easier to understand how the new physics has escaped the LHC searches. We do find that there are values of the parameters for which the cancellation between the bare mass and the radiative corrections is not severe and the LHC constraints are satisfied, including points where the top Yukawa coupling to the $125 \mathrm{GeV}$ Higgs is reduced and the cutoff scale for new physics is $5 \mathrm{TeV}$. We also find that the most natural regions of parameter space involve new couplings which run to fairly large values in the UV, which are still barely perturbative below the cutoff, but with Landau poles not too far above our cutoff. This could be a hint that the theory above the cutoff is strongly coupled or involves different weakly coupled degrees of freedom. Notably, a strongly coupled theory above the cutoff would involve 
light Goldstone bosons that could be searched for at colliders, leading to an even richer phenomenology. In some specific scenarios, it is even possible that some of these Goldstone bosons could be treated as the extra Higgs bosons introduced in our model [71-73].

Our results emphasize the importance of precision Higgs measurements, particularly direct measurements of the topHiggs coupling, as we find there is room in this model for significant deviation from 1 , and this value impacts the degree of fine-tuning and the expectation for the scale of new physics. Searches for additional Higgs bosons are also important in order to understand whether the naturalness paradigm that has played such a big role in theoretical physics could be realized in nature.

\section{ACKNOWLEDGMENTS}

S.E.H. acknowledges support by the NWO Vidi grant "Self-interacting asymmetric dark matter." A. N. is supported in part by the Kenneth Young Memorial Endowed Chair and in part by the DOE under Grant No. DE-SC0011637. D. W. is supported in part by a Burke faculty fellowship.

\section{APPENDIX A: SCALAR MASS EIGENSTATES}

In the limit where $\lambda_{i} \ll 1$ and $u, v_{1}, v_{2} \ll\left|A_{h}\right|$, the same neutral mass eigenstates are

$$
\begin{aligned}
& m_{1}^{2}=2 A_{h} u+\frac{2}{v_{1} v_{2}}\left(\left(\lambda_{1} v_{1}^{2}+\lambda_{4}\left(v_{1}+v_{2}\right)^{2}+\lambda_{2} v_{2}^{2}\right) v_{1} v_{2}-\left(\left(\lambda_{1}+2 \lambda_{4}-\lambda_{6}\right) v_{1}+\left(\lambda_{2}+2 \lambda_{4}-\lambda_{7}\right) v_{2}\right) u^{2}\left(v_{1}+v_{2}\right)\right) \\
& -\frac{1}{2 A_{h} u}\left(\left(\left(\lambda_{1}+\lambda_{4}\right)\left(3 \lambda_{1}-2 \lambda_{2}+\lambda_{4}-4 \lambda_{6}+4 \lambda_{7}\right) v_{1}^{2}+\left(\lambda_{2}+\lambda_{4}\right)\left(-2 \lambda_{1}+3 \lambda_{2}+\lambda_{4}+4 \lambda_{6}-4 \lambda_{7}\right) v_{2}^{2}\right) u^{2}\right. \\
& \left.+4\left(\lambda_{1}+\lambda_{4}\right)\left(\lambda_{2}+\lambda_{4}\right) v_{1}^{2} v_{2}^{2}\right)+\cdots, \\
& m_{2}^{2}=-2 A_{h} u+\frac{2}{v_{1} v_{2}}\left(\left(\left(\lambda_{1}+2 \lambda_{4}-\lambda_{6}\right) v_{1}-\left(\lambda_{2}+2 \lambda_{4}-\lambda_{7}\right) v_{2}\right) u^{2}\left(v_{1}-v_{2}\right)\right. \\
& \left.+\left(\left(\lambda_{1}+\lambda_{4}\right) v_{1}^{2}-2 \lambda_{4} v_{1} v_{2}+\left(\lambda_{2}+\lambda_{4}\right) v_{2}^{2}\right) v_{1} v_{2}\right)+\frac{1}{2 A_{h} u}\left(\left(\lambda_{1}+\lambda_{4}\right)\left(3 \lambda_{1}-2 \lambda_{2}+\lambda_{4}-4 \lambda_{6}+4 \lambda_{7}\right) v_{1}^{2}\right. \\
& \left.+\left(\lambda_{2}+\lambda_{4}\right)\left(-2 \lambda_{1}+3 \lambda_{2}+\lambda_{4}+4 \lambda_{6}-4 \lambda_{7}\right) v_{2}^{2}\right) u^{2}+4\left(\lambda_{1}+\lambda_{4}\right)\left(\lambda_{2}+\lambda_{4}\right) v_{1}^{2} v_{2}^{2}+\cdots \\
& m_{3}^{2}=4 u^{2}\left(\lambda_{1}+\lambda_{2}+\lambda_{3}+4 \lambda_{4}-\lambda_{6}-\lambda_{7}\right)-A_{h}+\left(\frac{v_{1} v_{2}}{u}+\frac{u v_{1}}{v_{2}}+\frac{u v_{2}}{v_{1}}\right) \cdots \text {. }
\end{aligned}
$$

\section{APPENDIX B: COLEMAN-WEINBERG POTENTIAL}

The scalar mass matrix, derived from Eq. (10), has the form $v^{\dagger} M_{s}^{2} v$ with $v=\left(h_{1}, h_{2}, \phi\right)$. Throughout this work, we require the scalar potential couplings to be $\lambda_{i}<1$. To understand the form of the effective potential, first consider the limit where $\lambda_{i}=0 . M_{s}^{2}$ is simply

$M_{s}^{2}=\frac{A_{h}}{3}\left(\begin{array}{ccc}-3 u v_{2} / v_{1} & \phi & h_{2} \\ \phi & -3 u v_{1} / v_{2} & h_{1} \\ h_{2}^{\dagger} & h_{1}^{\dagger} & -3 v_{1} v_{2} / u\end{array}\right)$.

In this limit, $M_{s}$ satisfies

$$
\frac{\partial}{\partial \theta} \operatorname{tr} M_{s}^{2}=0
$$

where $\theta=\left\{h_{1}, h_{2}, h_{1}^{\dagger}, h_{2}^{\dagger}, \phi\right\}$. This ensures that the effective potential has no quadratic diverging terms proportional to $A_{h}^{2}$. Moreover, the largest logarithmic terms are proportional to $A_{h}^{2}$.
We find that the quadratically diverging terms in the effective potential are

$$
\begin{aligned}
V_{\text {quadratic }}= & \frac{\Lambda^{2}}{32 \pi^{2}}\left(\lambda_{1}+2 \lambda_{4}+\frac{1}{2} \lambda_{5}+\frac{1}{2} \lambda_{6}\right) h_{1}^{\dagger} h_{1} \\
& +\frac{\Lambda^{2}}{32 \pi^{2}}\left(\lambda_{2}+2 \lambda_{4}+\frac{1}{2} \lambda_{5}+\frac{1}{2} \lambda_{7}\right) h_{2}^{\dagger} h_{2} \\
& +\frac{\Lambda^{2}}{32 \pi^{2}}\left(\lambda_{3}+\frac{\lambda_{6}}{2}+\frac{\lambda_{7}}{2}\right) \phi^{2}-\frac{3 \lambda^{2} \Lambda^{2}}{8 \pi^{2}} h_{2}^{\dagger} h_{2},
\end{aligned}
$$

where $\Lambda$ is the cutoff. We have omitted the contributions to the cosmological constant. The term proportional to $\lambda^{2}$ is generated by Eq. (25). The largest logarithmically divergent terms are

$$
\begin{aligned}
V_{\text {logarithm }}= & \frac{1}{64 \pi^{2}} \sum_{i=1}^{3} M_{i}^{4} \log \left[\frac{M_{i}^{2}}{\Lambda^{2}}\right] \\
& -\frac{3 \lambda^{4}}{16 \pi^{2}}\left(h_{2}^{\dagger} h_{2}\right)^{2} \log \left[\frac{\lambda^{2} h_{2}^{\dagger} h_{2}}{\Lambda^{2}}\right],
\end{aligned}
$$


where $M_{i}^{2}$ is defined by

$$
\begin{aligned}
M_{1}^{2}= & -\frac{A_{h} u}{2 v_{1} v_{2}}\left(v_{1}^{2}+v_{2}^{2}\right)-\left(\lambda_{1}+2 \lambda_{4}\right) v_{1}^{2}-\left(\lambda_{2}+2 \lambda_{4}\right) v_{2}^{2}+\frac{A_{h} u}{18 v_{1} v_{2}}\left(h_{1} h_{1}^{\dagger}+h_{1} h_{2}^{\dagger}+h_{2}^{\dagger} h_{1}+h_{2} h_{2}^{\dagger}\right) \\
& +\frac{A_{h}}{3} \phi-\frac{\lambda_{5}}{4}\left(h_{1}^{\dagger} h_{2}+h_{2}^{\dagger} h_{1}\right)+\frac{1}{4}\left(2 \lambda_{1}+4 \lambda_{4}+\lambda_{5}\right) h_{1}^{\dagger} h_{1}+\frac{1}{4}\left(2 \lambda_{2}+4 \lambda_{4}+\lambda_{5}\right) h_{2}^{\dagger} h_{2}+\frac{1}{4}\left(\lambda_{6}+\lambda_{7}\right) \phi^{2}, \\
M_{2}^{2}=- & \frac{A_{h} u}{2 v_{1} v_{2}}\left(v_{1}^{2}+v_{2}^{2}\right)-\left(\lambda_{1}+2 \lambda_{4}\right) v_{1}^{2}-\left(\lambda_{2}+2 \lambda_{4}\right) v_{2}^{2}+\frac{A_{h} u}{18 v_{1} v_{2}}\left(h_{1} h_{1}^{\dagger}-h_{1} h_{2}^{\dagger}-h_{2}^{\dagger} h_{1}+h_{2} h_{2}^{\dagger}\right) \\
& -\frac{A_{h}}{3} \phi+\frac{\lambda_{5}}{4}\left(h_{2}^{\dagger} h_{1}+h_{1}^{\dagger} h_{2}\right)+\frac{1}{4}\left(2 \lambda_{1}+4 \lambda_{4}+\lambda_{5}\right) h_{1}^{\dagger} h_{1}+\frac{1}{4}\left(2 \lambda_{2}+4 \lambda_{4}+\lambda_{5}\right) h_{2}^{\dagger} h_{2}+\frac{1}{4}\left(\lambda_{6}+\lambda_{7}\right) \phi^{2}, \\
& M_{3}^{2}=-\frac{A_{h} v_{1} v_{2}}{u}-\frac{A_{h} u}{9 v_{1} v_{2}}\left(h_{1} h_{1}^{\dagger}+h_{2} h_{2}^{\dagger}\right)+\frac{\lambda_{6}}{2} h_{1}^{\dagger} h_{1}+\frac{\lambda_{7}}{2} h_{2}^{\dagger} h_{2}+\lambda_{3} \phi^{2}-\left(\lambda_{6} v_{1}^{2}+\lambda_{7} v_{2}^{2}\right) .
\end{aligned}
$$

Here we have assumed that $u \gg \Lambda, \lambda_{i}<1$.

\section{APPENDIX C: RENORMALIZATION GROUP EQUATIONS}

Before canceling all the tadpole terms, the Higgs potential that we are using could be rewritten as

$$
\begin{aligned}
V= & \frac{\lambda_{1}^{\prime}}{2}\left(h_{1}^{\dagger} h_{1}\right)^{2}+\frac{\lambda_{2}^{\prime}}{2}\left(h_{2}^{\dagger} h_{2}\right)^{2}+\frac{\lambda_{3}^{\prime}}{24} \phi^{4}+\lambda_{4}^{\prime}\left(h_{1}^{\dagger} h_{1}\right)\left(h_{2}^{\dagger} h_{2}\right) \\
& +\lambda_{5}^{\prime}\left(h_{1}^{\dagger} h_{2}\right)\left(h_{2}^{\dagger} h_{1}\right)+\frac{\lambda_{6}^{\prime}}{2} \phi^{2}\left(h_{1}^{\dagger} h_{1}\right)+\frac{\lambda_{7}^{\prime}}{2} \phi^{2}\left(h_{2}^{\dagger} h_{2}\right) \\
& +A_{h}\left(\phi h_{1} h_{2}^{\dagger}+\phi h_{2} h_{1}^{\dagger}\right) .
\end{aligned}
$$

The primed couplings are related to the original couplings by

$$
\begin{gathered}
\lambda_{1}^{\prime}=2\left(\lambda_{1}+\lambda_{4}\right), \\
\lambda_{2}^{\prime}=2\left(\lambda_{2}+\lambda_{4}\right), \\
\lambda_{3}^{\prime}=24 \lambda_{3}, \\
\lambda_{4}^{\prime}=2 \lambda_{4}+\lambda_{5}, \\
\lambda_{5}^{\prime}=-\lambda_{5}, \\
\lambda_{6}^{\prime}=2 \lambda_{6}, \\
\lambda_{7}^{\prime}=2 \lambda_{7},
\end{gathered}
$$

and should remain of order one. The beta functions for the $2 \mathrm{HDM}$ are well-known [74]. We derive the contributions from the $\phi$ field as well as the renormalization group (RG) equation for $A_{h}$ from the Coleman-Weinberg potential. We checked our values for the contributions from the quartic couplings involving $\phi$ against [74]. Neglecting the weak couplings, we obtain

$$
\begin{aligned}
& 16 \pi^{2} \frac{d \lambda_{1}^{\prime}}{d t}=12 \lambda_{1}^{2}+4 \lambda_{4}^{\prime 2}+4 \lambda_{4}^{\prime} \lambda_{5}^{\prime}+2 \lambda_{5}^{\prime 2}+\lambda_{6}^{\prime 2}, \\
& 16 \pi^{2} \frac{d \lambda_{2}^{\prime}}{d t}= 12 \lambda_{2}^{2}+4 \lambda_{4}^{\prime 2}+4 \lambda_{4}^{\prime} \lambda_{5}^{\prime}+2 \lambda_{5}^{\prime 2}+\lambda_{7}^{\prime 2} \\
&+12 \lambda_{t}^{2} \lambda_{2}^{\prime}-12 \lambda_{t}^{4}, \\
& 16 \pi^{2} \frac{d \lambda_{3}^{\prime}}{d t}=6 \lambda_{3}^{\prime 2}+12\left(\lambda_{6}^{\prime 2}+\lambda_{7}^{\prime 2}\right), \\
& 16 \pi^{2} \frac{d \lambda_{4}^{\prime}}{d t}=\left(\lambda_{1}^{\prime}+\lambda_{2}^{\prime}\right)\left(6 \lambda_{4}^{\prime}+2 \lambda_{5}^{\prime}\right)+4 \lambda_{4}^{\prime 2}+2 \lambda_{5}^{\prime 2} \\
&+\lambda_{6}^{\prime} \lambda_{7}^{\prime}+6 \lambda_{t}^{2} \lambda_{4}^{\prime}, \\
& 16 \pi^{2} \frac{d \lambda_{5}^{\prime}}{d t}=2\left(\lambda_{1}^{\prime}+\lambda_{2}^{\prime}\right) \lambda_{5}^{\prime}+8 \lambda_{4}^{\prime} \lambda_{5}^{\prime}+4 \lambda_{5}^{\prime 2}+6 \lambda_{t}^{2} \lambda_{5}^{\prime}, \\
& 16 \pi^{2} \frac{d \lambda_{6}^{\prime}}{d t}=4 \lambda_{6}^{\prime 2}+6 \lambda_{1}^{\prime} \lambda_{6}^{\prime}+\lambda_{3}^{\prime} \lambda_{6}^{\prime}+4 \lambda_{4}^{\prime} \lambda_{7}^{\prime}+2 \lambda_{5}^{\prime} \lambda_{7}^{\prime}, \\
& 16 \pi^{2} \frac{d \lambda_{7}^{\prime}}{d t}=4 \lambda_{7}^{\prime 2}+6 \lambda_{2}^{\prime} \lambda_{7}^{\prime}+\lambda_{3}^{\prime} \lambda_{7}^{\prime}+4 \lambda_{4}^{\prime} \lambda_{6}^{\prime}+2 \lambda_{5}^{\prime} \lambda_{6}^{\prime}, \\
& 16 \pi^{2} \frac{d A_{h}}{d t}=8 A_{h}\left(\lambda_{4}^{\prime}+2 \lambda_{5}^{\prime}+\lambda_{6}^{\prime}+\lambda_{7}^{\prime}\right) .
\end{aligned}
$$

Here, $t=\log \mu$ (with $\mu$ the renormalization scale). Now, the RG equations for the Yukawa and gauge couplings (also including the strong coupling $g_{s}$ ) are also well-known and are [75]

$$
\begin{gathered}
16 \pi^{2} \frac{d \lambda_{t}}{d t}=\frac{9}{2} \lambda_{t}^{3}-8 g_{s}^{2} \lambda_{t}, \\
16 \pi^{2} \frac{d g_{s}}{d t}=-7 g_{s}^{3} .
\end{gathered}
$$


[1] G. Aad et al. (ATLAS Collaboration), Phys. Lett. B 716, 1 (2012).

[2] S. Chatrchyan et al. (CMS Collaboration), Phys. Lett. B 716, 30 (2012).

[3] K. G. Wilson, Phys. Rev. B 4, 3174 (1971).

[4] K. G. Wilson and J. B. Kogut, Phys. Rep. 12, 75 (1974).

[5] R. Barbieri and A. Strumia, arXiv:hep-ph/0007265.

[6] M. Ciuchini, E. Franco, S. Mishima, M. Pierini, L. Reina, and L. Silvestrini, Nucl. Part. Phys. Proc. 273-275, 2219 (2016).

[7] M. Schmaltz, Nucl. Phys. B, Proc. Suppl. 117, 40 (2003).

[8] E. Katz, A. E. Nelson, and D. G. E. Walker, J. High Energy Phys. 08 (2005) 074.

[9] A. G. Cohen, D. B. Kaplan, and A. E. Nelson, Phys. Lett. B 388, 588 (1996).

[10] Y. Hosotani, Phys. Lett. 126B, 309 (1983).

[11] A. T. Davies and A. McLachlan, Phys. Lett. B 200, 305 (1988).

[12] H. Hatanaka, T. Inami, and C. S. Lim, Mod. Phys. Lett. A 13, 2601 (1998).

[13] L. Randall and R. Sundrum, Phys. Rev. Lett. 83, 3370 (1999).

[14] N. Arkani-Hamed, A. G. Cohen, E. Katz, and A. E. Nelson, J. High Energy Phys. 07 (2002) 034.

[15] Z. Chacko, H.-S. Goh, and R. Harnik, Phys. Rev. Lett. 96, 231802 (2006).

[16] S. Dimopoulos and H. Georgi, Nucl. Phys. B193, 150 (1981).

[17] Current constraints on stops and heavy top quarks from ATLAS and CMS, https://atlas.web.cern.ch/Atlas/GROUPS/ PHYSICS/CombinedSummaryPlots/SUSY/ATLAS_SUSY_ Stop_tLSP/ATLAS_SUSY_Stop_tLSP.png, https://atlas.web .cern.ch/Atlas/GROUPS/PHYSICS/CombinedSummaryPlots/ EXOTICS/ATLAS_Exotics_Summary/ATLAS_Exotics_ Summary.png, https://twiki.cern.ch/twiki/pub/CMSPublic/ PhysicsResultsSUS/T6bbWW_limits_summary_cms.png, (accessed August 2016).

[18] M. Aaboud et al. (ATLAS Collaboration), Phys. Rev. D 97, 072003 (2018).

[19] A. M. Sirunyan et al. (CMS Collaboration), Phys. Rev. Lett. 120, 231801 (2018).

[20] G. Aad et al. (ATLAS and CMS Collaborations), J. High Energy Phys. 08 (2016) 045.

[21] We note the different sectors in the above equation could conceivably have different cutoffs. We restrict to Eq. (2) for simplicity.

[22] M. J. G. Veltman, Acta Phys. Pol. B 12, 437 (1981).

[23] M. Aaboud et al. (ATLAS Collaboration), J. High Energy Phys. 10 (2017) 182.

[24] M. Aaboud et al. (ATLAS Collaboration), Eur. Phys. J. C 78, 401 (2018).

[25] CMS Collaboration, CERN Report No. CMS-PAS-EXO17-011, 2017.

[26] R. Barbieri, L. J. Hall, and V. S. Rychkov, Phys. Rev. D 74, 015007 (2006).

[27] R. Barbieri and L. J. Hall, arXiv:hep-ph/0510243.

[28] I. Masina and M. Quiros, Phys. Rev. D 88, 093003 (2013).

[29] C. N. Karahan and B. Korutlu, Phys. Lett. B 732, 320 (2014).

[30] N. Chakrabarty and I. Chakraborty, arXiv:1801.05272.

[31] N. Darvishi and M. Krawczyk, Nucl. Phys. B926, 167 (2018).
[32] Y. G. Kim, K. Y. Lee, and S.-H. Nam, Phys. Lett. B 782, 316 (2018).

[33] A. Biswas and A. Lahiri, Proc. Sci., ICHEP20162016 (2016) 710 .

[34] N. Khan, Ph.D. thesis, Indian Inst. Tech., Indore, 2017.

[35] M. Chabab, M. C. Peyranere, and L. Rahili, Proc. Sci., ICHEP20162016 (2016) 794 [arXiv:1612.08770].

[36] A. D. Plascencia, J. High Energy Phys. 09 (2015) 026.

[37] A. Biswas and A. Lahiri, Phys. Rev. D 91, 115012 (2015).

[38] A. Kobakhidze and K. L. McDonald, J. High Energy Phys. 07 (2014) 155.

[39] I. Chakraborty and A. Kundu, Phys. Rev. D 90, 115017 (2014).

[40] I. Chakraborty and A. Kundu, Phys. Rev. D 89, 095032 (2014).

[41] O. Antipin, M. Mojaza, and F. Sannino, Phys. Rev. D 89, 085015 (2014).

[42] R. Jora, S. Nasri, and J. Schechter, Int. J. Mod. Phys. A 28, 1350036 (2013).

[43] F. Bazzocchi and M. Fabbrichesi, Phys. Rev. D 87, 036001 (2013).

[44] I. Chakraborty and A. Kundu, Phys. Rev. D 87, 055015 (2013).

[45] F. Bazzocchi and M. Fabbrichesi, Eur. Phys. J. C 73, 2303 (2013).

[46] J. A. Casas, J. R. Espinosa, and I. Hidalgo, J. High Energy Phys. 11 (2004) 057.

[47] X. P. Calmet, Eur. Phys. J. C 32, 121 (2003).

[48] A. Kundu and S. Raychaudhuri, Phys. Rev. D 53, 4042 (1996).

[49] E. Ma, Phys. Lett. B 732, 167 (2014).

[50] E. Ma, Int. J. Mod. Phys. A 16, 3099 (2001).

[51] S. R. Coleman and E. J. Weinberg, Phys. Rev. D 7, 1888 (1973).

[52] J. F. Gunion, H. E. Haber, G. L. Kane, and S. Dawson, Front. Phys. 80, 1 (2000).

[53] A. Schuessler and D. Zeppenfeld, in SUSY 2007 Proceedings, 15th International Conference on Supersymmetry and Unification of Fundamental Interactions, 2007, Karlsruhe, Germany (2007), pp. 236-239, http://inspirehep.net/record/ 978144.

[54] K. Betre, S. El Hedri, and D. G. E. Walker, Phys. Dark Universe 19, 46 (2018).

[55] Ya. B. Zeldovich, I. Y. Kobzarev, and L. B. Okun, Zh. Eksp. Teor. Fiz. 67, 3 (1974) [Sov. Phys. JETP 40, 1 (1974)].

[56] T. W. B. Kibble, J. Phys. A 9, 1387 (1976).

[57] A. Vilenkin, Phys. Rep. 121, 263 (1985).

[58] A. Drozd, B. Grzadkowski, J. F. Gunion, and Y. Jiang, J. High Energy Phys. 11 (2014) 105.

[59] M. Aaboud et al. (ATLAS Collaboration), Eur. Phys. J. C 78, 24 (2018).

[60] M. Aaboud et al. (ATLAS Collaboration), Eur. Phys. J. C 78, 293 (2018).

[61] M. Aaboud et al. (ATLAS Collaboration), J. High Energy Phys. 03 (2018) 174.

[62] CMS Collaboration, Report No. CMS-PAS-HIG-17-012, 2017.

[63] CMS Collaboration, Report No. CMS-PAS-HIG-16-025, 2016.

[64] ATLAS Collaboration, Report No. ATLAS-CONF-2016071, 2016. 
[65] ATLAS Collaboration, Report No. ATLAS-CONF-2016004, 2016.

[66] ATLAS Collaboration, Report No. ATLAS-CONF-2016089, 2016.

[67] A. Djouadi, Phys. Rep. 459, 1 (2008).

[68] H. Georgi and A. Pais, Phys. Rev. D 12, 508 (1975).

[69] N. Arkani-Hamed, A. G. Cohen, and H. Georgi, Phys. Lett. B 513, 232 (2001).

[70] A. Hasenfratz, C. Rebbi, and O. Witzel, Proc. Sci., EPS-HEP2017 (2017) 356 [arXiv:1710.02131].
[71] D. Barducci, S. De Curtis, M. Redi, and A. Tesi, arXiv: 1805.12578.

[72] O. Antipin and M. Redi, J. High Energy Phys. 12 (2015) 1 .

[73] C. Kilic and T. Okui, J. High Energy Phys. 04 (2010) 128.

[74] T. Alanne, K. Kainulainen, K. Tuominen, and V. Vaskonen, J. Cosmol. Astropart. Phys. 08 (2016) 057.

[75] M. E. Peskin, arXiv:hep-ph/9705479. 